\title{
Uma revisão das aproximações lineares para grandes amplitudes de oscilações do período de um pêndulo simples
}

\author{
A review of lienar approximations for large oscillation amplitudes of a simple pendulum
}

\author{
Flávio Silva Lopes ${ }^{* 1}$, Rogério Netto Suave ${ }^{1}$, Jose Alexandre Nogueira ${ }^{\dagger 1}$ \\ ${ }^{1}$ Universidade Federal do Espírito Santo, Departamento de Física, Centro de Ciências Exatas, Vitória, ES, Brasil
}

Recebido em 18 de Dezembro, 2017. Aceito em 24 de Fevereiro, 2018.

\begin{abstract}
Embora a aproximação $\sin \theta \approx \theta$ para determinação do período de um pêndulo simples seja muito bem conhecida e estudada desde o primeiro ano do ensino médio, ela tem bons resultados somente para pequenas amplitudes de oscilações. Já as aproximações para grandes amplitudes de oscilações são praticamente desconhecidas pela grande maioria dos professores e alunos do ensino médio e superior. Então, neste trabalho, nós fazemos uma revisão das principais sugestões de aproximações lineares para grandes amplitudes de oscilações para o cálculo do período de um pêndulo simples e uma comparação entre seus resultados.
\end{abstract}

Palavras-chave: Pêndulo simples, aproximações lineares, grandes amplitudes.

\begin{abstract}
Although the $\sin \theta \approx \theta$ approximation to calculate the period of a simple pendulum is well-known and studied from the first year of high school, it only has good results for small oscillation amplitudes. Now, the approximations for large oscillation amplitudes are almost completely unknown by the vast majority of theachers and students of high school and of undergraduate. Hence, in this work, we review the main approximation suggestions for large oscillation amplitudes to calculate the period of a simple pendulum and we make a comparison between their results, too.
\end{abstract}

Keywords: simple pendulum, linear approximations, large amplitudes.

\section{Introdução}

O estudo de um pêndulo simples é comumente abordado na totalidade dos livros textos de física do ensino médio [1 3] e dos cursos de graduação [4 6]. O pêndulo simples também tem servido como paradigma no estudo de problemas lineares e não lineares $[7-[10]$. Ainda, é muito comum o uso do pêndulo simples na literatura para exemplificar determindos assuntos que se está abordando (como meio para atingir um objetivo), como faz J. Clement 11 para demonstrar que os estudantes têm conceitos primitivos mal compreendidos, ou fazem R. N. Suave e J. A. Nogueira [12 para discutir os cuidados que se deve tomar em fazer aproximações e desprezar termos considerados de menor ordem. Embora, aparentemente simples, o estudo de um pêndulo simples pode ser um problema muito rico, como pode ser visto nas referências 13, 14. Pelo fato de ser um experimento de relativamente fácil execução, ele é muito usado para determinação do módulo da aceleração da gravidade $g$.

O cálculo para obtenção do período exato do pêndulo simples é algo complexo (para alunos das disciplinas de física básica) e seu resultado não pode ser dado em termos de funções elementares. Por esse motivo, nos livros

*Endereço atual: Pontifícia Universidade Católica do Rio de Janeiro (PUC-Rio)

†Endereço de correspondência: jose.nogueira@ufes.br textos do ensino médio, sem exceções, e das disciplinas básicas de física dos cursos de graduação, quase sem exceções, o período de um pêndulo simples é determindado apenas considerando-se pequenas amplitudes de oscilações, onde a aproximação linear $\operatorname{sen} \theta \approx \theta$ é empregada. Nessa aproximação, o pêndulo simples é muito usado para ilustrar o movimento harmônico simples (MHS). Contudo, a princípio, tal aproximação fornece somente bons resultados para amplitudes de oscilações menores que $10^{0}\left(\theta_{M}<10^{0}\right)$. Isso faz com que os resultados obtidos nos experimentos para a determinação do período de um pêndulo simples possam não ser satisfatórios, pois os experimentos precisam ser realizados com muito cuidado, isto é, para ângulos de pequenas amplitudes, o que dificulta a obtenção de medidas precisas do período. Então, existe a necessidade de que o cálculo teórico para o período do pêndulo simples possa ser empregado não só para pequenas amplitudes mas também para grandes amplitudes. Entretanto, o cálculo teórico do período exato do pêndulo simples depende de uma integral elíptica do primeiro tipo, a qual não pode ser realizada analiticamente, levando-nos a recorrer a tabelas ou softwares, como o Mathematica ou o MatLab. 
A fim de contornar esse problema, alguns autores ${ }^{1}$ têm sugerido diferentes tipos de aproximações que podem ser empregadas para o cálculo do período de um pêndulo simples para grandes amplitudes (fórmulas para o cálculo do período). As aproximações lienares, em geral são bem simples, e foram propostas por L. H. Cadwell \& E. R. Boyco [15], W. P. Ganley [16], M. I. Molina [17], R. B. Kidd \& S. L. Fogg 18, 19, R. R. Parwani 20] e G. E. Hite 21]. Diversos outros tipos de aproximações foram porpostadas por outros autores, tais como, logarítmicas por F. M. S. Lima \& P. Arun 22] e por Xue De-Sheng et al [23], analíticas por Beléndez et al $24-26]$ e M. Turkyilmazoglu [27], aritmética-geomémtrica por C. G. Carvalhaes \& P. Suppes [28], assintótica por A. Cromer [29], combinação da logarítmica e da assintótica por F. M. S. Lima [30], expansão delta linear por P. Amore et al [31], além de outras.

O objetivo deste trabalho é realizar uma revisão detalhada das aproximações lineares propostas por M. I. Molina 17], R. B. Kidd \& S. L. Fogg, [18] e R. R. Parwani (W. P. Ganley) 16 20], com a sugestão de que as mesmas sejam trabalhadas como atividades complementares nas disciplinas de física básica, principalmente, nos cursos de bacharelado e licenciatura de Física. Embora, existam outras propostadas de aproximações lineares com melhores precisões, como por exemplo a de Hite [21], a escolha das propostas de Molina, Kidd-Fogg e Parwani é porque elas são simples e têm como base conceitos físicos elementares.

Também reforçamos a sugestão, já feita por outros autores 1821 , que a fórmula usual, $T=2 \pi \sqrt{\frac{l}{g}}$, para o cálculo do período de um pêndulo simples seja trocada pela de Kidd-Fogg, $T=2 \pi \sqrt{\frac{l}{g \cos \left(\frac{\theta_{M}}{2}\right)}}$, nos livros texto de disciplinas básicas de física dos cursos de graduação, bem como também nos do ensino médio, além de manuais de laboratóros. Entretanto, sugerimos que a introdução dessa fórmula seja feita através da abordagem proposta por L. E. Millet [19], devido a sua simplicidade e por ela depender apenas de relações trigonométricas elementares que fazem parte do conteúdo do primeiro ano do ensino médio.

O trabalho está organizado da seguinte forma: na Seção 2. uma rápida revisão da aproximação $\sin \theta \approx \theta$ é realizada. Nas Seções 3, 4 e 5 as propostas de aproximação de Molina, Kidd-Fogg e Ganley-Parwani são, respectivamente, apresentadas. Na Subseção 4.1, a abordagem de Millet para a obtenção da fórmula para aproximação de Kidd-Fogg é mostrada. Na Seção 6, é realizada uma comparação das precisões obtidas pelas aproximações anteriores. Por fim, na Seção 7, são feitas as considerações finais e apresentadas nossas conclusões. O trabalho conta também com os apêndices A e B, onde o cálculo do período exato e a aproximação de Bernoulli são revistos, respectivamente.

\footnotetext{
${ }^{1}$ Pedimos desculpas aos autores que, sem intenção, deixamos de citar aqui.
}

\section{Aproximação linear $\sin \theta \approx \theta$}

A Figura 1 mostra as forças que agem sobre a massa $m$ do pêndulo. Aplicando a Segunda Lei de Newton na direção tangencial tem-se

$$
m l \frac{d^{2} \theta}{d \theta^{2}}=-m g \sin \theta
$$

onde $m$ é a massa do pêndulo e $l$ seu comprimento. Se a amplitude de oscilação $\theta_{M}$ é muito pequena, $\sin \theta \approx \theta$, pois $\theta \leq \theta_{M}$. Então, a Eq. (1) fica

$$
\frac{d^{2} \theta}{d \theta^{2}}=-\frac{g}{l} \theta
$$

Nesta aproximação, a massa $m$ do pêndulo simples realiza movimento harmônico simples de período

$$
T_{0}=2 \pi \sqrt{\frac{l}{g}} .
$$

A Figura 2 mostra ${ }^{2}$ o erro relativd ${ }^{3}$ entre o valor do período calculado exato (veja Apêndice A) e o da aproximação $\sin \theta \approx \theta$ em função da amplitude de oscilação.

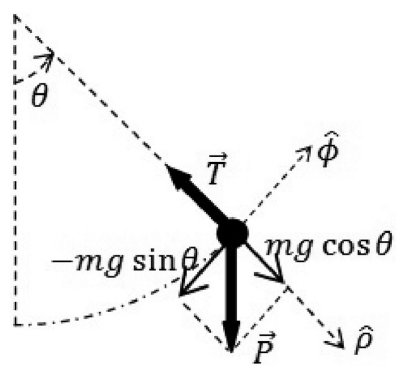

Figura 1: Decomposição das forças sobre os eixos radial e tangencial.

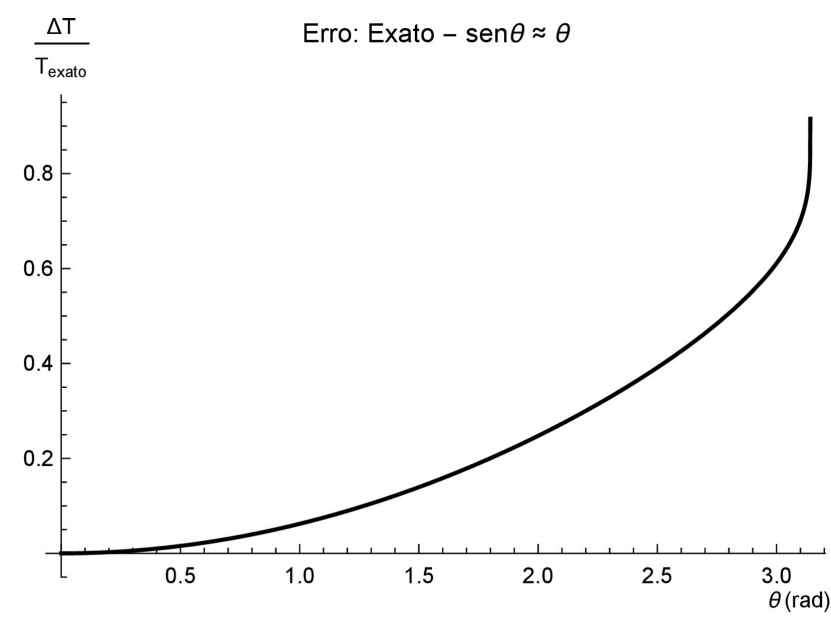

Figura 2: Erro relativo entre o período exato e a aproximação $\sin \theta \approx \theta$.

\footnotetext{
$\overline{2}$ Os resultados numéricos e os gráficos apresentados neste trabalho foram obtidos pelo uso do software Mathematica 11.1.1, Licença número 3523-8649.

${ }^{3} \Delta T:=\left|\frac{T_{0}-T_{\text {exato }}}{T_{\text {exato }}}\right|$.
} 
Os erros percentuais para alguns valores da amplitude podem ser vistos na Tabela 1.

\section{Aproximação linear de Molina, 1997}

A proposta de M. I. Molina 17 é aproximar a componente tangencial, não linear, da força resultante agindo na massa $m$ do pêndulo (veja Figura 1) por uma linear, isto é,

$$
\sin \theta \approx F\left(\theta_{M}\right) \theta
$$

A função $F\left(\theta_{M}\right)$ é, então, um coeficiente que tende a compensar a não linearidade desta força. Embora, exista uma liberdade na escolha de $F\left(\theta_{M}\right)$, ela deve satisfazer alguns requisitos:

1. Se a amplitude de oscilação é muito pequena, isto é, $\theta_{M}$ é muito pequeno, a aproximação $\sin \theta \approx \theta$ é adequada, o que implica que $F\left(\theta_{M}\right) \approx 1$ quando $\theta_{M}$ é muito pequend 4 .

2. Quando a massa $m$ do pêndulo se encontra inicialmente na vertical, acima do ponto de fixação do fio, isto é, $\theta_{M}=\pi$, a componente tangencial da força resultante é nuld 5 Portanto, quando $\theta_{M}=\pi \rightarrow F\left(\theta_{M}\right)=0$

3. Uma vez que há simetria em relação ao eixo vertical, o módulo da componente tangencial da força resultante é igual para $\theta_{M}$ e $-\theta_{M}$. Portanto, a função $F\left(\theta_{M}\right)$ deve ser par, isto é, $F\left(\theta_{M}\right)=F\left(-\theta_{M}\right)$. Note que a inversão de sentido da componente tangencial da força já está contido em $\theta\left(-\theta_{M} \leq \theta \leq\right.$ $\left.\theta_{M}\right)$.

A função proposta por Molina, que satisfaz os requisitos acima, é

$$
F\left(\theta_{M}\right)=\left(\frac{\sin \theta_{M}}{\theta_{M}}\right)^{\alpha}
$$

onde $\alpha$ é um parâmetro a ser determinado. A escolha de como determinar o parâmetro $\alpha$ não é única, sendo que diferentes escolhas devem levar a diferentes resultados para $\alpha$.

Tabela 1: Erro: exato e $\sin \theta \approx \theta$

\begin{tabular}{lc}
\hline$\theta_{M}$ & Erro \% \\
\hline $10^{0}$ & 0,1904 \\
$30^{0}$ & 1,711 \\
$60^{0}$ & 6,819 \\
$90^{0}$ & 15,27 \\
$114,6^{0}$ & 24,75 \\
\hline
\end{tabular}

\footnotetext{
${ }^{4}$ É claro que, neste caso, $\theta_{M}$ puder ser considerado muito pequeno depende da precisão desejada para o período.

${ }^{5}$ Note que a componente tangencial da força resultante também é nula no ponto mais baixo da trajetória. Contudo, nesse caso, $\theta=0$ na aprxomação, o que anula a componente tangencial da força resultante.
}

Usando a proposta acima, a equação de movimento do pêndulo simples fica

$$
\frac{d^{2} \theta}{d \theta^{2}}=-\frac{g}{l}\left(\frac{\sin \theta_{M}}{\theta_{M}}\right)^{\alpha} \theta .
$$

Da Eq. (6) acima, o período pode ser facilmente encontrado como

$$
T=T_{0}\left(\frac{\sin \theta_{M}}{\theta_{M}}\right)^{-\frac{\alpha}{2}} .
$$

Para determinação do parâmetro $\alpha$, Molina impôs que a primeira correção para o período extato do pêndulo simples, isto é, o coeficiente do termo de ordem $\theta^{2}$ da aproximação não linear de Bernoulli (veja Apêndice B),

$$
T_{\text {Bernoulli }}=2 \pi \sqrt{\frac{l}{g}}\left(1+\frac{\theta_{M}^{2}}{16}\right),
$$

coincida com o coeficiente do termo de mesma ordem obtido da expansão em série de Taylor do resultado acima,

$$
\begin{aligned}
T & =T_{0} \frac{1}{\theta_{M}}\left(\theta_{M}-\frac{\theta_{M}^{3}}{3 !}+\ldots\right)^{-\frac{\alpha}{2}} \\
& =T_{0}\left(1+\frac{\alpha}{12} \theta_{M}^{2}+\ldots\right) .
\end{aligned}
$$

Portanto,

$$
\alpha=\frac{3}{4} .
$$

Assim, a aproximação linear (para grandes amplitudes de oscilações) proposta por Molina fica

$$
T_{\text {Molina }}=2 \pi \sqrt{\frac{l}{g}}\left(\frac{\sin \theta_{M}}{\theta_{M}}\right)^{-\frac{3}{8}} .
$$

A Figura 3 mostra os resultados exato e o da aproximação de Molina para o período do pêndulo simples. Como pode ser visto, o valor do período calculado pela

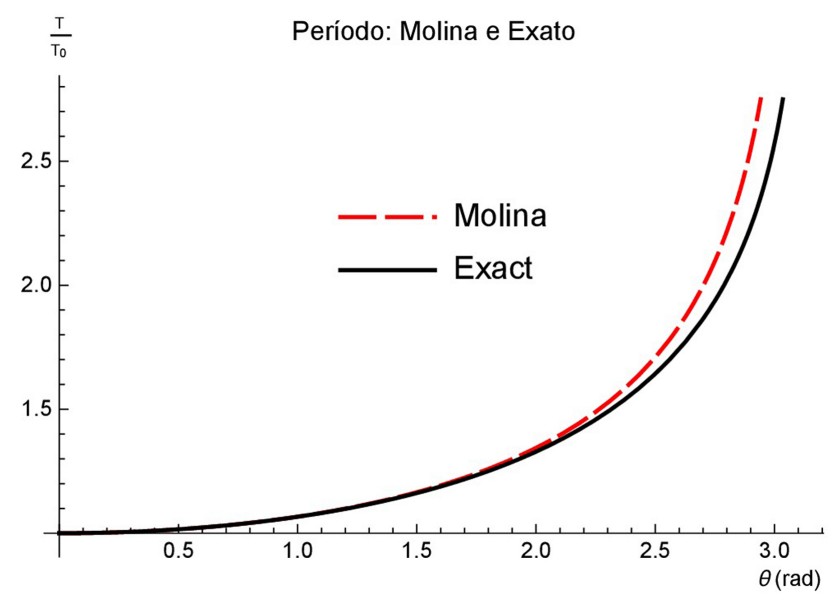

Figura 3: Comparação do período exato com o da aproximação de Molina. 
aproximação de Molina é sempre maior que o do cálculo exato, e há uma excelente concordância até amplitudes de $90^{\circ}$ (menores que $1 \%$ ). Note que o resultado do cálculo do período para a aproximação $\sin \theta \approx \theta$ está sobre o eixo horizontal. Então, as distâncias do eixo horizontal e da linha trancejada até a linha contínua fornecem uma fácil visualização de comparação entre os erros das aproximações $\sin \theta \approx \theta$ e a de Molina. Como pode-se notar, os resultados são significativamente melhores para a aproximação de Molina. Por fim, como acontece para o cálculo exato do período, nesta aproximação o período também diverge para $\theta_{M}=\pi$.

A Figura 4 mostra o erro relativo entre os valores exato e o da aproximação de Molina em função da amplitude de oscilação. Os erros percentuais para alguns valores da amplitude podem ser vistos na Tabela 2 . Até cerca de 1,1 radianos $\left(63^{0}\right)$, a curva da Figura 4 está sobre o eixo da abscissa, o que indica erros muito pequenos (menores que $0,1 \%)$. Para 2,0 radianos $\left(114,6^{0}\right)$ o erro é pouco maior que $1 \%$.

\section{Aproximação linear de Kidd-Fogg, 2002}

A proposta de R. B. Kidd and S. L. Fogg [18 tem como base a analogia entre o período de um sistema massa-mola e o da aproximação para $\sin \theta \approx \theta$ do pêndulo simples. $\mathrm{Na}$ aproximação $\sin \theta \approx \theta$, a equação de movimento para a massa $m$ do pêndulo simples, Eq. (2), é analoga a de

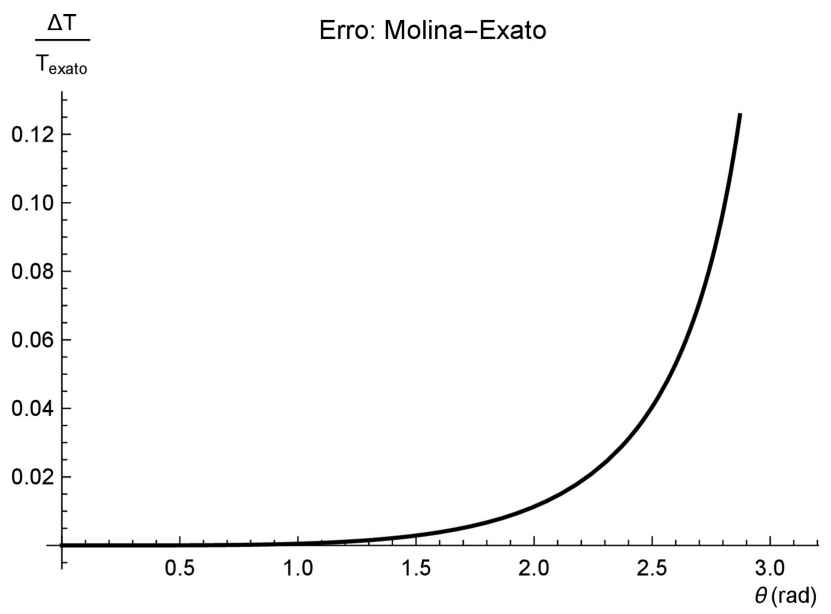

Figura 4: Erro relativo entre o período exato e a aproximação de Molina. um sistema massa-mola em 1 dimensão (1-dim),

$$
\frac{d^{2} x}{d t^{2}}=-\frac{k}{M} x
$$

$\operatorname{com} \frac{k}{M} \equiv \frac{g}{l}$. Ambas as equações são a de um oscilador harmônico,

$$
\frac{d^{2} X}{d t^{2}}=-\omega^{2} X
$$

cujo período é $T=\frac{2 \pi}{\omega}$ e $\omega$ é uma constante.

No caso do sistema massa-mola em 1-dim a força restauradora, responsável pelo movimento oscilatório, é

$$
F=-k x \text {. }
$$

Então,

$$
k=\left|\frac{d F}{d x}\right| .
$$

Assim, o período para o sistema massa-mola pode ser escrito comb 6

$$
T=2 \pi \sqrt{\frac{M}{\left|\frac{d F}{d x}\right|}}
$$

Por analogia, para um pêndulo simples têm-se que

$$
T=2 \pi \sqrt{\frac{m}{\left|\frac{d F}{d s}\right|}}
$$

ond $\AA^{7} s=l \theta$. Para o pêndulo simples a força restauradora é dada por

$$
F=-m g \sin \theta
$$

Então,

$$
\left|\frac{d F}{d s}\right|=\frac{m g}{l} \cos \theta
$$

que, exceto para o caso da aproximação $\sin \theta \approx \theta$, não é uma constante.

A proposta de kidd-Fogg é considerar que o período de um pêndulo simples possa ser dado em termos de

$$
\left|\frac{d F}{d \theta}\right|=m g \cos \beta
$$

${ }^{6}$ Considere uma partícula de massa $m$ sob a ação de um potencial convexo $V(x)$, cujo ponto de mínimo esteja em $x_{0}$, isto é, $x_{0}$ seja ponto de equílibrio estável. A expansão de $V(x)$ em torno de $x_{0}$ fica,

$V(x)=V\left(x_{0}\right)+\frac{d V\left(x_{0}\right)}{d x}\left(x-x_{0}\right)+\frac{1}{2} \frac{d^{2} V\left(x_{0}\right)}{d x^{2}}\left(x-x_{0}\right)^{2}+\mathcal{O}\left(x-x_{0}\right)^{3}$.

Para pequenos deslocamentos em torno de $x_{0}$, os termos de $\mathcal{O}\left(x-x_{0}\right)^{3}$ são desprezíveis. Então, lembrando que $F\left(x_{0}\right)=$ $-\left.\frac{d V}{d x}\right|_{x_{0}}=0$, escolhendo $V\left(x_{0}\right)=0$ e redefindo $X:=x-x_{0}$,

$$
V(X)=\left.\frac{1}{2} \frac{d^{2} V}{d X^{2}}\right|_{0} X^{2}
$$

que é o potencial de um oscilador harmônico. Portanto, a partícula realizará um movimento harmônico simples cujo período é

$$
T=\sqrt{\frac{m}{\left.\frac{d^{2} V}{d X^{2}}\right|_{0}} .}
$$

${ }^{7}$ Note que $k$ tem dimensão de $\left[\right.$ força] $[\mathrm{L}]^{-1}$. 
onde $\beta$ é um ângulo fixo (uma constante) entre 0 e $\theta_{M}$. Assim,

$$
T_{K F}=2 \pi \sqrt{\frac{l}{g \cos \beta}}=T_{0} \sqrt{\frac{1}{\cos \beta}} .
$$

É fácil ver que, quando o parâmetro $\beta=0, T_{K F}=T_{0}$, mas quando $\beta=\frac{\pi}{2}, T_{K F}$ diverge. Então, $0 \leq \beta<\frac{\pi}{2}$.

A escolha do parâmetro $\beta$ é, a princípio, arbitária. Para determinar o parâmetro $\beta$, Kidd e Fogg, da mesma forma como Molina fez, impuseram que a expansão de $T_{K F}$ até $\mathcal{O}(\beta)^{2}$ coincida com a aproximação de Bernoulli, Eq. 8 . Assim,

$$
\begin{aligned}
T_{0} \sqrt{\frac{1}{\cos \beta}} & =T_{0}\left(1-\frac{\beta^{2}}{2}+\ldots\right)^{-\frac{1}{2}} \\
& \approx T_{0}\left(1+\frac{\beta^{2}}{4}+\ldots\right)
\end{aligned}
$$

e, consequentemente,

$$
\beta=\frac{\theta_{M}}{2} .
$$

Usando o resultado acima na Eq. 21, obtém-se

$$
T_{K F}=2 \pi \sqrt{\frac{l}{g \cos \left(\frac{\theta_{M}}{2}\right)}} .
$$

A Figura 5 mostra os resultados exato e o da aproximação de Kidd-Fogg para o período do pêndulo simples. Como pode ser visto, como ocorre na aproximação de Molina, os valores para a aproximação de Kidd-Fogg são sempre maiores que os do cálculo exato.

A Figura 6 mostra o erro relativo entre os valores exato e o da aproximação de Kidd-Fogg em função da amplitude de oscilação. Os erros percentuais para alguns valores da amplitude podem ser vistos na Tabela 3. Como pode-se notar, embora os resultados sejam significativamente melhores que os da aproximação $\sin \theta \approx \theta$, os da aproximação de Molina são melhores. Os erros percentuais da aproximação de Kidd-Fogg são cerca de 2 vezes maiores que os da aproximação de Molina.

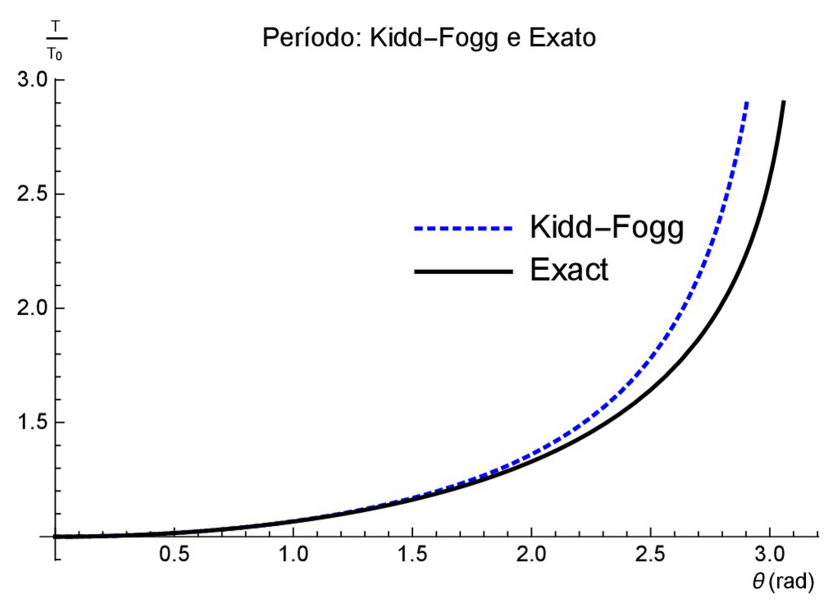

Figura 5: Comparação do período exato com o da aproximação de Kidd-Fogg.

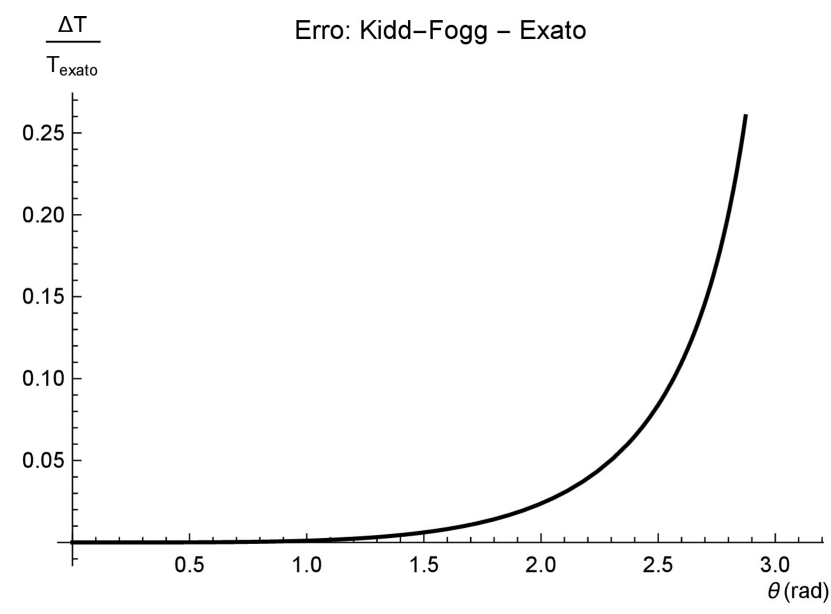

Figura 6: Erro relativo entre o período exato e a aproximação de Kidd-Fogg.

Tabela 3: Erro: exato e Kidd-Fogg.

\begin{tabular}{cc}
\hline$\theta_{M}$ & Erro \% \\
\hline $10^{0}$ & $9,085 \times 10^{-5}$ \\
$30^{0}$ & $7,512 \times 10^{-3}$ \\
$60^{0}$ & $1,293 \times 10^{-1}$ \\
$90^{0}$ & $7,512 \times 10^{-1}$ \\
$114,6^{0}$ & 2,3736 \\
\hline
\end{tabular}

\subsection{Millet, 2003}

L. E. Millet 19 reobteve o resultado da aproximação de Kidd-Fogg de forma muito simples e elegante. Para isso, ele usou a bem conhecida relação trigonométrica

$$
\sin \theta=2 \cos \left(\frac{\theta}{2}\right) \sin \left(\frac{\theta}{2}\right) .
$$

Para amplitudes de pequenos ângulos $\theta_{M}, \sin \left(\frac{\theta}{2}\right) \approx \frac{\theta}{2}$ e $\cos \left(\frac{\theta}{2}\right) \approx 1$, e a aproximação $\sin \theta \approx \theta$ é obtida. Agora, se $\theta_{M}$ não é pequeno o bastante, com o propósito de manter a linearidade da aproximação, sin $\left(\frac{\theta}{2}\right)$ é trocado por $\frac{\theta}{2}$ e $\cos \left(\frac{\theta}{2}\right)$ por $\cos \left(\frac{\theta_{M}}{2}\right)$. Assim,

$$
\sin \theta \approx \cos \left(\frac{\theta_{M}}{2}\right) \theta .
$$

A troca de $\cos \left(\frac{\theta}{2}\right)$ por $\cos \left(\frac{\theta_{M}}{2}\right)$ pode ser vista não só como para manter a lineridade da aproximação, mas também como uma forma de compensar a não linearidade de $\sin \theta$. A acurácia (validade) dessa troca fica mais evidente quando se nota ${ }^{8}$ que $\frac{\theta}{2}$ é sempre um pouco maior que $\sin \left(\frac{\theta}{2}\right)$, enquanto $\cos \left(\frac{\theta}{2}\right)$ é sempre menor que $\frac{\theta}{2}$. Então, o produto deles é uma forma aproximada de compensar o acréscimo da troca $\sin \left(\frac{\theta}{2}\right) \approx \frac{\theta}{2}$. Desta forma, a equação de movimento do pêndulo, Eq. (1), fica

$$
\frac{d^{2} \theta}{d \theta^{2}}=-\frac{g}{l} \cos \left(\frac{\theta_{M}}{2}\right) \theta,
$$

${ }^{8} \operatorname{Para} \theta \neq 0$. 
a qual conduz ao período $T=T_{0} \frac{1}{\sqrt{\cos \left(\frac{\theta_{M}}{2}\right)}}$.

O leitor pode estar se perguntando por que usar a relação 25. A resposta é simples: pode-se justificar o uso da relação (25), notando que $\sin \theta$ é mais linear no intervalo $\left[0, \frac{\theta_{M}}{2}\right]$ do que no intervalo $\left[0, \theta_{M}\right]$.

\section{Aproximação linear de Ganley-Parwani, 1985-2003}

A proposta de R. R. Parwani [20] é escrever a equação de movimento do pêndulo simples na forma sugestiva

$$
\frac{d^{2} \theta}{d t^{2}}=-\frac{g}{l}\left(\frac{\sin \theta}{\theta}\right) \theta
$$

No limite de pequenos ângulos $\frac{\sin \theta}{\theta}$ tende a uma constante, mais precisamente a 1. Então, uma tentativa de aproximação para o período é tratar $\frac{\sin \theta}{\theta}$ como uma constante, trocando a variável $\theta$ por um parâmetro de valor fixo, isto é, $\frac{\sin \theta}{\theta} \rightarrow \frac{\sin \beta}{\beta}$. O período fica assim dado por

$$
T=2 \pi \sqrt{\frac{l}{g}}\left(\frac{\sin \beta}{\beta}\right)^{\frac{1}{2}} .
$$

Parwani também observou que, por uma simples análise dimensional, o período do pêndulo simples deve ser da forma

$$
T \approx T_{0} F\left(\theta_{M}\right) .
$$

Comparando as Eqs. 29) e 30 , obtém-se

$$
F\left(\theta_{M}\right) \approx\left(\frac{\sin \beta}{\beta}\right)^{\frac{1}{2}}
$$

Portanto, o parâmetro $\beta$ deve depender de alguma forma da amplitude de oscilação $\theta_{M}$. Assim, como fizeram Molina e Kidd-Fogg, para determinar o parâmetro $\beta$, Parwani impôs que a expansão do período da Eq. (29) em potências de $\beta$ (pois, para $\beta$ pequeno $T \rightarrow T_{0}$ ),

$$
T=T_{0}\left(1-\frac{\beta^{2}}{3 !}+\frac{\beta^{4}}{5 !}+\ldots\right),
$$

coincida com a aproximaçãode Bernoulli, Eq. (8). Consequentemente,

$$
\begin{gathered}
\frac{\theta_{M}^{2}}{16}=\frac{\beta^{2}}{12}, \\
\beta=\frac{\sqrt{3}}{2} \theta_{M} .
\end{gathered}
$$

Por fim, a aproximação de Ganley-Parwan $\sqrt[9]{\text { para o pe- }}$ ríodo do pêndulo simples fica

$$
T_{G P}=2 \pi \sqrt{\frac{l}{g}}\left[\frac{\sin \left(\frac{\sqrt{3}}{2} \theta_{M}\right)}{\frac{\sqrt{3}}{2} \theta_{M}}\right]^{-\frac{1}{2}}
$$

Tabela 4: Erro: exato e Ganley-Parwani.

\begin{tabular}{lc}
\hline$\theta_{M}$ & Erro \% \\
\hline $10^{0}$ & $6,092 \times 10^{-6}$ \\
$30^{0}$ & $5,274 \times 10^{-4}$ \\
$60^{0}$ & $1,055 \times 10^{-2}$ \\
$90^{0}$ & $7,772 \times 10^{-2}$ \\
$114,6^{0}$ & $3,168 \times 10^{-1}$ \\
\hline
\end{tabular}

A Figura 7 mostra os resultados exato e o da aproximação de Ganley-Parwani para o período do pêndulo simples. Como pode ser visto, ao contrário das aproximações de Molina e Kidd-Fogg, os valores para a aproximação de Ganley-Parwani são sempre menores que os do cálculo exato. Ainda, é possível notar que ambas as curvas, praticamente, coincidem até cerca de 1,9 radianos $\left(108^{0}\right)$.

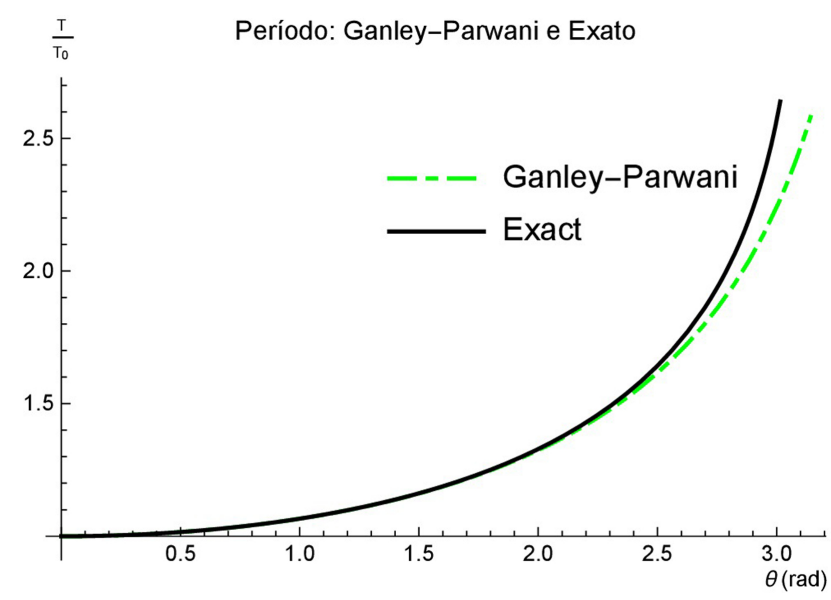

Figura 7: Comparação do período exato com o da aproximação de Ganley-Parwani.

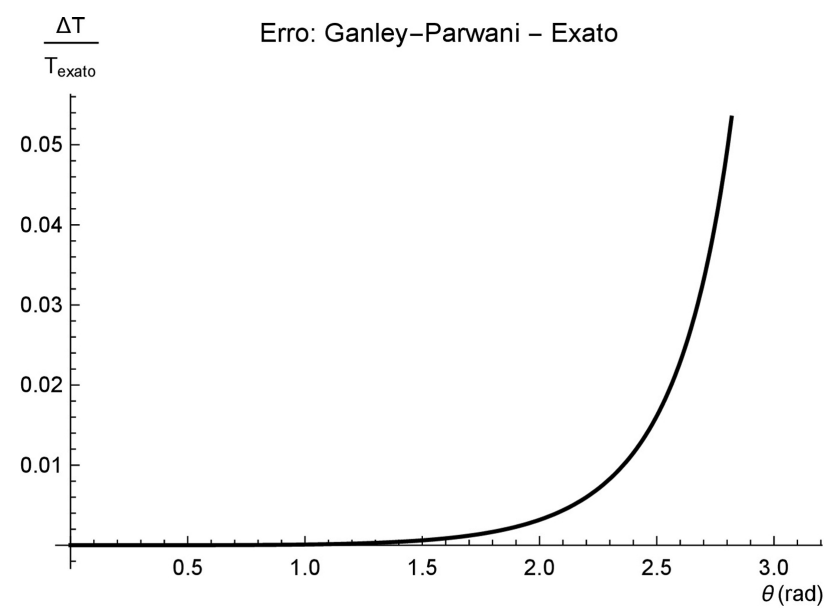

Figura 8: Erro relativo entre o período exato e a aproximação de Ganley-Parwani.

\footnotetext{
${ }^{9} \mathrm{~A}$ denominação desta aproximação de Ganley-Parwani é porque, embora por um método diferente, W. P. Ganley já a havia obtido em [16].
} 
A Figura 8 mostra o erro relativo entre os valores exato e o da aproximação de Ganley-Parwani em função da amplitude de oscilação. Os erros percentuais para alguns valores da amplitude podem ser vistos na Tabela 4 . Como pode-se notar, os resultados são melhores que os da aproximação os de Molina. O erro percentual da aproximação de Molina é cerca de uma magnitude superior ao da aproximação de Ganley-Parwani para a amplitude de $10^{0}$ (mais precisamente, 7 vezes maior), e diminui para menos 5 vezes para $90^{\circ}$.

\section{Comparações dos resultados}

Na Figura 9 estão representados, em um único gráfico, as curvas dos períodos obtidos pelo cálculo exato e pelas aproximações $\sin \theta \approx \theta$, de Molina, de Kidd-Fogg e de Ganley-Parwani. Uma rápida olhada permite afirmar que a aproximação de Ganley-Parwani tem os melhores resultados. Isso fica evidente na Figura 10 que mostra os erros relativos percentuais das aproximações. Qualquer reta horizontal sempre cortará a curva da aproximação de Kidd-Fogg em primeiro lugar, depois a de Molina e, por último a de Ganley-Parwani. As intersecções da linha tracejada horizontal e as curvas mostram para amplitudes de que ângulos o erro é de $1 \%$.

$\mathrm{Na}$ tabela 5 estão reunidos alguns valores dos erros das aproximações lineares discutidas anteriormente.

É tentador avaliar os erros cometidos nas aproximações com a correção de maior ordem $\left(\theta_{M}^{4}\right)$ da expansão em série de potências da integral elíptica, Eq. (A.11), porém,

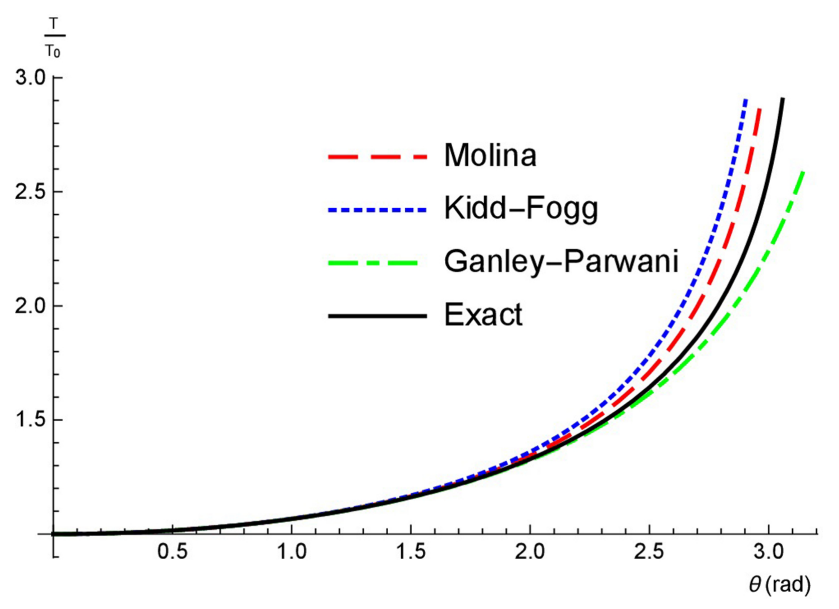

Figura 9: Gráfico comparativo dos Períodos.

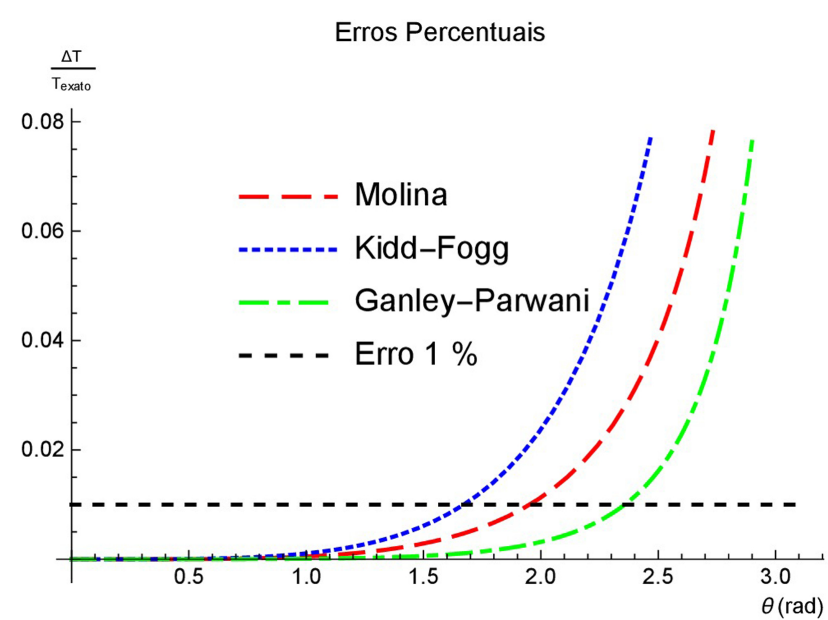

Figura 10: Erros percentuais das aproximações.

isso seria um erro, pois para amplitudes de ângulos maiores que 1 radiano não existe garantia de que a expansão seja assintótica já a partir do segundo ou terceiro termos, isto é, que o terceiro termo seja menor que o segundo.

\section{Considerações Finais e Conclusão}

Neste trabalho, foi feita uma revisão detalhada das aproximações lineares para o cálculo do período de um pêndulo simples propostas por Molina, Kidd \& Fogg e Parwani. Embora, a aproximação de Kidd-Fogg não seja a que conduz aos melhores resultados (veja Tabela 5), a maneira muito simples de obté-la mostrada por Millet faz com ela seja indicada como uma possibilidade de se extender a fórmula do cálculo do período de um pêndulo simples para amplitudes de grandes ângulos nos livros textos das disciplinas de física básica dos cursos de graduação e, até mesmo, de ensino médio.

Dos resultados obtidos, pode-se ver que, enquanto a aproximação $\sin \theta \approx \theta$ atinge um erro superior a $15 \%$ para uma amplitude de ângulo de $90^{\circ}$, as aproximações de Molina, Kidd-Fogg e Ganley-Parwani são menores que $1 \%$. Isto mostra o quanto são eficazes essas aproximações, embora sejam simples e um tanto ingênuas. Da Figura 10 e da tabela 5 pode-se concluir que a aproximação de Ganley-Parwani é a que fornece os melhores resultados, com erros percentuais cerca de dez vezes menores que os obtidos pelas aproximações de Molina e Kidd-Fogg.

Tabela 5: Erros percentuais: $\left|\frac{T-T_{\text {exato }}}{T_{\text {exato }}}\right| \times 100$.

\begin{tabular}{lcccc}
\hline & \multicolumn{4}{c}{ Erro $\%$} \\
\cline { 2 - 5 }$\theta_{M}$ & $\sin \theta \approx \theta$ & Molina & Kidd-Fogg & Ganley-Parwani \\
\hline $10^{0}$ & 0,1904 & $4,240 \times 10^{-5}$ & $9,085 \times 10^{-5}$ & $6,092 \times 10^{-6}$ \\
$30^{0}$ & 1,711 & $3,510 \times 10^{-3}$ & $7,512 \times 10^{-3}$ & $5,274 \times 10^{-4}$ \\
$60^{0}$ & 6,819 & $6,066 \times 10^{-2}$ & $1,293 \times 10^{-1}$ & $1,055 \times 10^{-2}$ \\
$90^{0}$ & 15,27 & $3,547 \times 10^{-1}$ & $7,512 \times 10^{-1}$ & $7,772 \times 10^{-2}$ \\
$114,6^{0}$ & 24,75 & 1,129 & 2,3736 & $3,168 \times 10^{-1}$ \\
\hline
\end{tabular}


O leitor pode estar questionando da real necessidade de usar outra aproximação do que a $\sin \theta \approx \theta$, visto que o erro é de apenas $1,7 \%$ para um ângulo de amplitude de oscilações de $30^{\circ}$, e, apesar de $30^{\circ}$ ser considerado um grande ângulo de amplitude, para um período de $T_{0}=1 \mathrm{~s}$ o erro é de apenas $17 \mathrm{~ms} 32$. Embora, pequeno esse erro é factível de ser medido com a tecnologia atual, mesmo em laboratórios de ensino 22 .

Outro ponto que o leitor pode estar questionando é que com a disponibilidade atual de acesso a softwares e aplicativos que permitem encontrar em pouco instantes os valores de funções especiais e integrais, tal como da integral elíptica completa de primeiro tipo, pode parecer justificar a falta de necessidade de se estudar aproximações para o período de um pêndulo simples. Contudo, essa pode ser uma conclusão ingênua e precipitada, uma vez que é o contornar do desenvolvimento de cálculos "complexos" para a obtenção do período exato que justifica o uso de aproximações para grandes amplitudes de oscilações cujas obtenções de algumas delas são simples e acessível para os alunos nos primeiros anos da graduação, ou até mesmo para os alunos do ensino médio.

\section{Material suplementar}

O seguinte material suplementar está disponível online: Apêndice

\section{Referências}

[1] C.S. Calçada e J.L. Sampaio, Física Clássica - Volume Dinâmica Estática e Volume Óptica-Ondas (Atual Editora, São Paulo, 2003).

[2] V.B. Newton, D.R. Helou e J.B. Gaulter, Tópicos de Física (Editora Saraiva, São Paulo, 2008), $18^{\mathrm{a}}$ ed., v. 2, p. 174.

[3] F. Ramalho Jr., G.F. Nicolau e P.A.S. de Toledo, Os Fundamentos da Física (Editora Moderna Plus, São Paulo, 2009), v. 2, p. 398.

[4] H. Moysés Nussenzveig, Curso de Física Básica (Editora Edgard Blucher, São Paulo, 2013), 5a ed., v. 2, p. 48.

[5] R. Resnick, D. Hallliday e K.S. Krane, Física (Editora LTC, Rio de Janeiro, 2003), $5^{\text {a }}$ ed. , v. 2, p. 94.

[6] H.D. Young e R.A. Freedman, Física (Editora AddisonWesley, São Paulo, 2003), 50ª ed., v. 2, p. 49.

[7] S.T. Thornton and J.B. Marion, Classical Dynamics of Particles and Systems (Thomsom Books, New York, 2003), 5th ed., p. 162.

[8] G.R. Fowles and G.L. Cassiday, Analytical Mechanics (Saunders College Publishing, New York, 2004), 7th ed., p. 110.

[9] Walter Greiner, Classical Mechanics - Systems of Particles and Hamiltonian Dynamics (Springer-Verlag, New York, 2010), 2nd ed., p. 22 e 537.

[10] G.B. Arfken and H.J. Weber, Mathematical Methods for Physicists (Academic Press, San Diego, 1995), 4th ed., p. 331 .

[11] J. Clement, Am. J. Phys. 50, 66 (1982).
[12] R.N. Suave e J.A. Nogueira, Rev. Brs. Ens. Fis. 38, e2501 (2016).

[13] R.A. Nelson and M.G. Olsson, Am. J. Phys. 54, 112 (1986).

[14] G.L. Baker and J.A. Blackburn, The Pendulum: A Case Study in Physics (Oxford University Press, Oxford, 2005).

[15] L.H. Cadwell and E.R. Boyco, Am. J. Phys. 59, 979 (1991).

[16] W.P. Ganley, Am. J. Phys. 53, 73 (1985).

[17] M.I. Molina, Phys. Teach. 35, 489 (1997).

[18] R.B. Kidd and S.L. Fogg, Phys. Teach. 40, 81 (2002).

[19] L.E. Millet, Phys. Teach. 41, 162 (2003).

[20] R.R. Parwani, Eur. J. Phys. 25, 37 (2004).

[21] G.E. Hite, Phys. Teach. 43, 290 (2005).

[22] F.M.S. Lima and P. Arun, Am. J. Phys. 74, 892 (2006).

[23] Xue De-Sheng, Zhou Zhao and Gao Mei-Zhen, Chin. Phys. Lett. 29, 044601 (2012).

[24] A. Beléndez, A. Hernández, A. Márquez, T. Beléndez and C. Neipp, Eur. J. Phys. 27, 539 (2006).

[25] A. Beléndez, J.J. Rodes, T. Beléndez and A. Hernández, Eur. J. Phys. 30, L25 (2009).

[26] A. Beléndez, J. Franés, M. Ortuño, S. Gallego and J.G. Bernabeu, Eur. J. Phys. 31, L65 (2010).

[27] M. Turkyilmazoglu, Eur. J. Phys. 31, 1007 (2010).

[28] C.G. Carvalhaes and P. Suppes, Am. J. Phys. 76, 1150 (2008).

[29] A. Cromer, Am. J. Phys. 63, 112 (1995).

[30] F.M.S. Lima, Eur. J. Phys. 29, 1091 (2008).

[31] P. Amore, M.C. Valdovinos, G. Ornelas and S.Z. Barajas, Rev. Mex. Fis E 53, 106 (2007).

[32] N. Aggarwal, N. Verma and P. Arun, Eur. J. Phys. 26, 517 (2005). 